Summary: This study reports on the production of chitosan fibers and 3-D fiber meshes for the use as tissue engineering scaffolds. Both structures were produced by means of a wet spinning technique. Maximum strain at break and tensile strength of the developed fibers were found to be $8.5 \%$ and $204.9 \mathrm{MPa}$, respectively. After $14 \mathrm{~d}$ of immersion in simulated body fluid (SBF), scanning electron microscopy (SEM), energy dispersive spectroscopy (EDS), and inductively coupled plasma emission (ICP) spectroscopy analyses showed that a bioactive Ca-P layer was formed on the surface of the fibers, meaning that they exhibit a bioactive behavior. The samples showed around $120 \%$ max. swelling in physiological conditions. The pore sizes of 3-D chitosan fiber mesh scaffolds were observed to be in the range of $100-500 \mu \mathrm{m}$ by SEM. The equilibrium-swelling ratio of the developed scaffolds was found to be around $170 \%(\mathrm{w} / \mathrm{w})$ in $\mathrm{NaCl}$ solution at $37^{\circ} \mathrm{C}$. Besides that, the limit swelling strain was less than $30 \%$, as obtained by mechanical spectroscopy measurements in the same conditions. The viscoelastic properties of the scaffolds were also evaluated by both creep and dynamic mechanical tests. By means of using short-term MEM extraction test, both types of structures (fibers and scaffolds) were found to be non-cytotoxic to fibroblasts. Furthermore, osteoblasts directly cultured over chitosan fiber mesh scaffolds presented good morphology and no inhibition of cell proliferation could be observed.

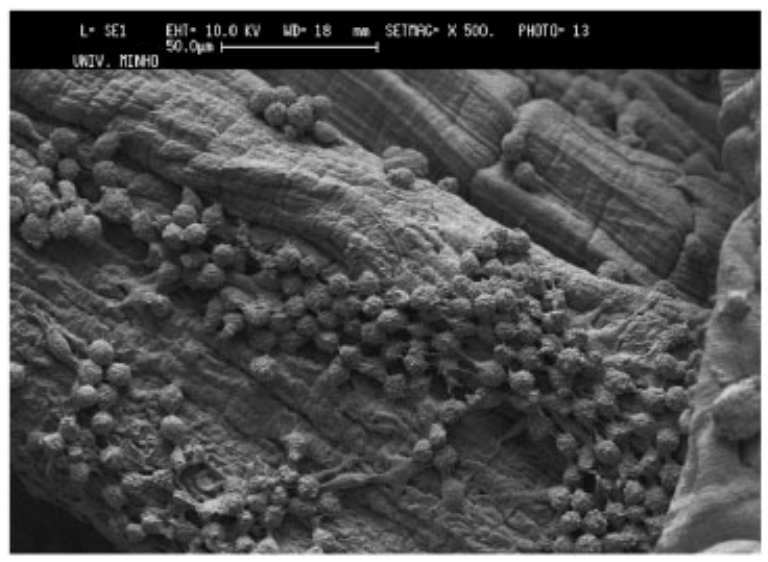

Osteoblast-like cells proliferating over chitosan based fibers after $7 \mathrm{~d}$ of culture.

\title{
Production and Characterization of Chitosan Fibers and 3-D Fiber Mesh Scaffolds for Tissue Engineering Applications
}

\author{
Kadriye Tuzlakoglu, ${ }^{1,2}$ Catarina M. Alves, ${ }^{1,2}$ Joao F. Mano, ${ }^{1,2}$ Rui L. Reis ${ }^{* 1,2}$ \\ ${ }^{1}$ Department of Polymer Engineering, University of Minho, Campus de Azurém, 4800-058 Guimarães, Portugal \\ Fax: +351 253604492; E-mail: rgreis@ dep.uminho.pt \\ 23B's Research Group-Biomaterials, Biodegradables and Biomimetics, University of Minho, Campus de Gualtar, \\ 4710-057 Braga, Portugal
}

Received: November 12, 2003; Revised: May 3, 2004; Accepted: May 24, 2004; DOI: 10.1002/mabi.200300100

Keywords: biodegradable; chitosan; cytotoxicity; fibers; tissue engineering

\section{Introduction}

Tissue engineering needs the development of better polymeric scaffolds, which among other characteristics should be biodegradable and biocompatible. The scaffolds can be applied simultaneously as a carrier matrix for bioactive agents and as a support to seed primary undifferentiated cells. A number of natural ${ }^{[1,2]}$ and synthetic polymers are currently in use as tissue scaffolds. ${ }^{[3]}$ Depending on the applications, scaffolds can be presented in different forms, such as for instances membranes, and several types of 3-D architectures. Within the last years, non-woven mesh-like scaffolds have been started to be used in tissue engineering applications due to their highly porous structures. Polyglycolide (PGA) ${ }^{[4,5]}$ polylactide/polyglycolide (PLA/ PGA), ${ }^{[6]}$ and chitosan meshes ${ }^{[7]}$ have been used as a scaffold for different tissue engineering applications.

Chitosan, a $(1 \rightarrow 4)$-2-amino-2-deoxy- $\beta$-D-glucose, is derived from chitin, which is found in the exo-skeleton of shellfish like shrimp or crabs. Because of its stable, crystalline structure, chitosan is normally insoluble in aqueous solutions above $\mathrm{pH}$ 7. However, in dilute acids, 
the free amino groups are protonated and the molecule becomes fully soluble below $\mathrm{pH} 5{ }^{[8]}$ Viscous solutions can be formed into fibers in different coagulation solution with high $\mathrm{pH}$, such as aq. $\mathrm{NaOH},{ }^{[9]}$ aq. $\mathrm{KOH},{ }^{[10]}$ aq. $\mathrm{NaOH}-40 \%$ methanol, ${ }^{[11]}$ and aq. $\mathrm{NaOH}-\mathrm{NaSO}_{4}$ (or AcONa) mixture. ${ }^{[12]}$ Chitosan fiber forming properties have been investigated by several researchers. ${ }^{[13]}$ A number of methods have been tried in order to produce chitosan fibers with good mechanical properties. ${ }^{[14-16]}$

Chitosan is a well known natural polymer that is biodegradable, biocompatible and non-toxic. ${ }^{[17]}$ It has been shown ${ }^{[18,19]}$ to aggressively bind to a variety of mammalian and microbial cells. Binding on cells and biodegradability of chitosan may lead to a variety of biomedical applications such as wound dressing, ${ }^{[20]}$ carriers for drug delivery systems ${ }^{[21,22]}$ and space filling implants. ${ }^{[23,24]}$ Recent studies of chitosan confirm the utility of chitosan for promoting the bone growth. ${ }^{[25,26]}$. Muzzarelli et al. ${ }^{[25]}$ reported the formation of mineralized bone-like tissue in osseous defects in rats, sheep and dogs when using chitosan plugs.

In the present study, chitosan fibers were produced for being used subsequently to process tissue engineering scaffolds. The developed scaffolds should ideally combine an adequate porous structure with proper mechanical and degradation properties. Furthermore, this study aimed for the analysis of this materials cytotoxicity as a first screening of its biocompatibility by means of using mouse fibroblast (L929) and human osteoblast (SAOS-2) cell lines.

\section{Experimental Part}

\section{Materials}

Medium molecular weight chitosan with an $85 \%$ degree of deacetylation was obtained from Sigma-Aldrich. All other chemicals were analytical grade and used as received.

\section{Methods}

\section{Chitosan Fiber Preparation}

Chitosan fibers were produced by a wet spinning method as described elsewhere. ${ }^{[12]}$ In a typical procedure, chitosan was dissolved in aq. $2 \%(\mathrm{v} / \mathrm{v})$ acetic acid in 5\% (w/v) concentration by stirring at room temperature overnight. The solution was diluted with methanol to reach $3 \%$ (w/v) final solution concentration. Glycerol at $2.5 \%(\mathrm{w} / \mathrm{w})$ concentration was added into this solution as a plasticizer. The solution was filtered through a cloth filter and put in an ultrasonic bath to remove the air bubbles. The solution was injected into a coagulation bath at $40{ }^{\circ} \mathrm{C}$ containing a mixture of $30 \% 0.5 \mathrm{M} \mathrm{Na}_{2} \mathrm{SO}_{4}, 10 \% 1 \mathrm{M}$ $\mathrm{NaOH}$ and $60 \%$ distilled water. The fibers were kept in this coagulation medium for one day and washed several times with distilled water. They were then suspended in aq. $30 \%$ methanol for $4-5 \mathrm{~h}$ and then in aq. 50\% methanol overnight. The chitosan filaments were wounded on the cylindrical support.
The fibers were dried at room temperature/humidity conditions for one day.

\section{Chitosan Fiber Meshes Preparation}

A similar production method was used to produce 3-D chitosan fiber meshes. However, a different drying method and a thinner needle were used. After fibers were formed in the coagulation medium at room temperature, they were kept in this solution overnight. The fibers were then washed several times with distilled water before being suspended in $50 \%$ methanol for $1 \mathrm{~h}$. Then they were suspended in $100 \%$ methanol for $3 \mathrm{~h}$ and put in the oven at $55^{\circ} \mathrm{C}$ for drying in a mould.

\section{Characterization}

\section{Chitosan Fiber}

Scanning Electron Microscopy: The morphology of fibers was visualized by Scanning Electron Microscopy (SEM) in a Leica Cambridge S360 microscope. Samples were sputter coated with gold.

Mechanical Properties: Tensile tests were performed on a Universal Test Machine (Lyloyd Instruments, LR \% K Serensworth Fareham, England), with a $500 \mathrm{~N}$ load cell, using a crosshead speed of $20 \mathrm{~mm} / \mathrm{min}$ and a gauge length of $20 \mathrm{~mm}$. Average values of tensile strength and maximum strain were determined after repeating the test five times.

Swelling Tests: Swelling behaviors of fibers have been investigated in $0.154 \mathrm{M} \mathrm{NaCl}$ aqueous isotonic saline solution at $\mathrm{pH}=7.4$. Fibers with different glycerol ratios were used, in order to observe the effect of glycerol on the swelling ratio. The samples were immersed in the solution, taken out from it at various time intervals, and then weighted with an electronic balance. The experiment was continued until samples reached equilibrium. The swelling degree was calculated according to the following equation

$$
\% S=\left(m_{\mathrm{w}}-m_{\mathrm{i}}\right) / m_{\mathrm{i}} \times 100
$$

where $\% S$ is the percentage of swelling, $m_{\mathrm{w}}$ is the weight of the wet sample after immersion in the $\mathrm{NaCl}$ solution.

Bioactivity Tests: Chitosan fibers were immersed in $15 \mathrm{ml}$ simulated body fluid (SBF) in a plastic bottle at $37^{\circ} \mathrm{C}$. SBF solution was proposed initially by Kokubo et al. ${ }^{[27]}$, presents an ionic composition similar to human blood plasma, and has been widely used ${ }^{[28,29]}$ to test the bioactivity of different materials in vitro. After various periods of incubation (3, 7, 14, $30 \mathrm{~d}$ ) in SBF, samples were removed from the solution, washed carefully with water and dried at room temperature in a desiccator. The apatite formation on the fiber surface of a calcium-phosphate film was visualized by SEM (Leica Cambridge S360). EDS spectra were obtained after the observation. Changes in concentration of calcium and phosphorus of SBF solution due to soaking of fibers were measured using inductively-coupled plasma emission (ICP) spectroscopy.

Cytotoxicity - Short Term MEM Extraction Test using Fibroblast Cells: To establish the cytotoxic rate of leachables, a MEM Extract test, a cytotoxicity test laid down in European and International standards (ISO/EN 109935 Guidelines) was 
performed. ${ }^{[30]}$ For this assay, a cell line of mouse lung fibroblasts (L929) was selected. Growth to confluency was performed in a controlled atmosphere $\left(37^{\circ} \mathrm{C}, 5 \% \mathrm{CO}_{2}, 100 \%\right.$ humidity $)$ using Dulbecco's Modified Eagle's Medium (DMEM, Sigma), supplemented with 10\% Foetal Bovine Serum (FBS, Biochrome) and $1 \%$ antibiotic/antimicotic solution (Sigma). In order to achieve $80 \%$ of confluency, cells seeded in cell culture polystyrene plates $\left(10000\right.$ cells $\left./ \mathrm{cm}^{2}\right)$ were incubated for $24 \mathrm{~h}$.

According to ISO/EN 109935 Guidelines, the surface area of the different samples was determined and immersion in the correspondent cell culture media volume was performed $\left(37^{\circ} \mathrm{C}, 60 \mathrm{rpm}\right)$. The same procedure was carried out for a negative control Ultra High Molecular Weight Polyethylene (UHMWPE), and positive control Latex Rubber. After $24 \mathrm{~h}$, fibroblasts were morphologically analyzed, and the culture medium was replaced by the extraction one. Test samples and controls were incubated in triplicate $(n=3)$. After 24,48 and $72 \mathrm{~h}$ testing, the reaction of cells to extracts was evaluated by means of light microscopy and compared to the positive control and negative control. A score for confluency of monolayer, degree of floating cells and changes in cellular morphology was calculated based on the scores posted on Table 1. In the end of the test, the cell number was measured and the percentage of cell growth inhibition determined. By means of combining the different quantitative and qualitative parameters scores, the obtained cytotoxic index defines if samples fail or pass the cytotoxicity test (used scores can be seen in Table 2).

\section{Chitosan Fiber Meshes}

Scanning Electron Microscopy: The mesh structure of chitosan fibers was analyzed by Scanning Electron Microscopy (Leica Cambridge S360) again after coating with gold.

Mechanical Properties: Short-time swelling, creep and dynamic mechanical analysis (DMA) experiments were carried out in a DMA7e Perkin-Elmer apparatus, in the compression mode, using parallel plates with $10 \mathrm{~mm}$ diameter. The analyzed samples, with cylindrical shape, were typically $2 \mathrm{~mm}$ high. All experiments were performed while the samples were immersed in an isotonic saline solution. In order to implement such runs, a metallic cylindrical vessel was fitted into the inner part of the furnace of the apparatus. The vessel was filled with isotonic saline solution. The calibration of the force was performed with the top plate immersed, in order to take into account the impulsion effect of the solution. Before the runs have been carried out on the samples, the temperature of the bath was stabilized at $37^{\circ} \mathrm{C}$, using the own temperature sensor of the apparatus.
The short-time monitoring of the swelling of the studied mesh was carried out using the thermal mechanical analysis (TMA) mode of the Perkin-Elmer dynamic mechanical analysis (DMA) apparatus. Briefly, the temperature of the dry sample is kept at $37^{\circ} \mathrm{C}$ in an external furnace, near the DMA equipment. The sample is quickly positioned between the plates and the furnace with the bath is immediately raised up. The height of the sample starts to be recorded less than $10 \mathrm{~s}$ after the sample contacts the liquid. During the experiment a small stress of $10 \mathrm{~Pa}$ is imposed to the sample by the top plate, in order to maintain the contact between the sample and the plates.

In the creep experiments, previously swelled samples are positioned between the plates and immersed until the temperature equilibrates at $37^{\circ} \mathrm{C}$. The creep stress was $0.1 \mathrm{MPa}$. A program of creep/recovery was used, where the strain, $\varepsilon$, was measured against time.

DMA experiments were performed in the immersed samples (with equilibrated water content) at $37^{\circ} \mathrm{C}$. The real (storage modulus), $E^{\prime}$, and the imaginary component (loss modulus), $E^{\prime \prime}$, of the complex modulus, $E^{*}=E^{\prime}+\mathrm{i} E^{\prime \prime}$ (with $\mathrm{i}=(-1)^{1 / 2}$ ), were recorded against frequency, that varied between 0.5 and $10 \mathrm{~Hz}$. A static stress of $36 \mathrm{kPa}$ and a dynamic stress of $30 \mathrm{kPa}$ were used in such experiments.

Swelling Tests: The swelling ratios of the samples were calculated using the same experimental set-up and method that was described above.

Cytotoxicity and Biocompatibility - Direct Contact Test with Osteoblast-Like Cells: Cytotoxicity assay was performed for chitosan fiber meshes by following the same methodology as described above for chitosan fiber preparation.

In order to study cell morphology, attachment and proliferation onto the chitosan fiber meshes scaffolds, a human osteoblasts SAOS-2 cell line was selected. Former described culture conditions were also used for osteoblast-like cells. Cells cultured in DME Media (Sigma), enriched with $10 \%$ FBS (Biochrome) and 1\% antibiotic/antimicotic solution (Sigma) were seeded directly over samples in a concentration of $3.3 \times 10^{4} \mathrm{cell} / \mathrm{ml}$. Incubation was performed at $37{ }^{\circ} \mathrm{C}\left(5 \% \mathrm{CO}_{2}, 100 \%\right.$ humidity) for $24 \mathrm{~h}$ and $7 \mathrm{~d}$. Tissue culture grade polystyrene (TCPS) discs were used as control and replicates were always prepared. After each incubation period, samples were washed with phosphate buffer saline (PBS, Sigma) solution and fixed in glutaraldehyde $2.5 \%(\mathrm{v} / \mathrm{v})$. For scanning electronic microscopy (SEM) observation, samples were previously dehydrated in crescent alcohol concentrations $(50 \%, 70 \%, 90 \%$ and $100 \%)$, air-dried and sputter coated with gold.

Table 1. Quantitative and qualitative scores used in the cytotoxicity tests.

\begin{tabular}{|c|c|c|c|c|}
\hline \multirow[t]{2}{*}{ Score } & Confluency & Floating cells & \multirow[t]{2}{*}{ Change of cellular morphology } & Inhibition of cell growth \\
\hline & $\%$ & $\%$ & & $\%$ \\
\hline 0 & 100 & 0 & No changes during test period & $0-10$ \\
\hline 1 & $90-100$ & $0-5$ & Slight changes, few cells affected & $10-30$ \\
\hline 2 & $60-90$ & $5-10$ & Mild changes, some cells round/spindle shaped & $30-50$ \\
\hline 3 & $30-60$ & $10-20$ & Moderate changes, many cells round/spindle & $50-70$ \\
\hline 4 & $0-30$ & $>20$ & Severe changes, about all cells show morphological changes & $70-100$ \\
\hline
\end{tabular}


Table 2. Different cytotoxicity indexes used to classify the reactivity of tested samples.

\begin{tabular}{lll}
\hline Cytotoxic response & Reactivity & Pass/Fail \\
\hline $0-1$ & None & Pass \\
$1-3$ & Slight & Pass \\
$3-5$ & Mild & Retest \\
$5-7$ & Moderate & Fail \\
$7-8$ & Severe & Fail
\end{tabular}

\section{Results and Discussion}

\section{Chitosan Fibers}

\section{Scanning Electron Microscopy}

Figure 1a and $\mathrm{b}$ present SEM images of the developed chitosan fibers. As it can be seen here in, chitosan fibers have been oriented in one direction due to the stretching process. Additionally, the images show that the fibers have a smooth and uniform striated surface even though using drying elements and rolling up the fiber on cylindrical support following a methanol drying bath may damage a fiber surface. For instance, Knaul et al. ${ }^{[15]}$ have reported the effects of different drying elements on chitosan fiber surfaces.

a)

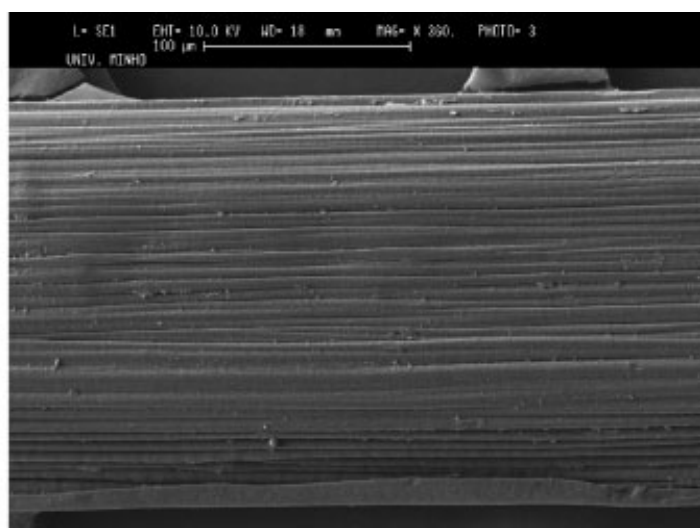

b)

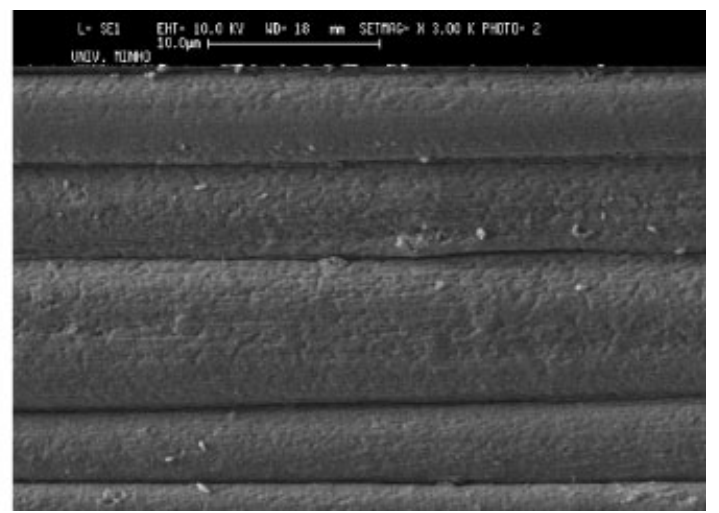

Figure 1. SEM micrograph of chitosan fibers a) $\times 360$, b) $\times 3000$ magnification.

\section{Mechanical Properties}

With regard to tensile tests, maximum strain at break and tensile strength of fibers were found to be $8.5 \pm 2.3 \%$ and $204.9 \pm 9.7 \mathrm{MPa}$, respectively.

\section{Swelling Tests}

Figure 2 shows the swelling behavior of the produced fibers. As it can be seen in the graph, the fibers showed a rapid swelling in the first $30 \mathrm{~min}$, and then they gradually tend to equilibrium that is achieved in about $120 \mathrm{~min}$.

\section{Bioactivity Tests}

Figure 3a, b, c present SEM micrographs of chitosan fibers before (Figure 3a) and after a bioactivity test for $30 \mathrm{~d}$ (Figure 3b, c). In Figure 3a it is possible to observe the typical oriented morphology of the fiber. Similar morphology was observed in the first days of immersion in SBF. However, after $14 \mathrm{~d}$ of immersion, a number of nuclei started to form on the surface of the fibers, that by EDS analysis were found to correspond to the formation of calcium phosphate (Ca-P) (Data not shown). This nuclei have grown with increasing immersion times and after $30 \mathrm{~d}$, a compact and well-defined Ca-P layer could be observed, as presented in Figure $3 \mathrm{~b}$ and $3 \mathrm{c}$. For higher magnifications (Figure 3c) this layer tended to the typical so-called cauliflower-like morphology of Ca-P films. A commercial polyamide fiber was used as a control and for this case no Ca-P film could be observed even after $30 \mathrm{~d}$ of immersion in SBF (Figure 4). This clearly indicates the bioactivity of the developed fibers, confirming what has been observed by authors such as Muzzarelli in vivo. ${ }^{[25]}$ This result is supported by EDS and ICP analysis (Figure 5 and 6, respectively). $\mathrm{Ca}$ and $\mathrm{P}$ elements in the formed layers can be clearly seen in EDS spectra. In addition, ICP results showed that there was a decreasing of $\mathrm{Ca}$ and $\mathrm{P}$ concentration in the

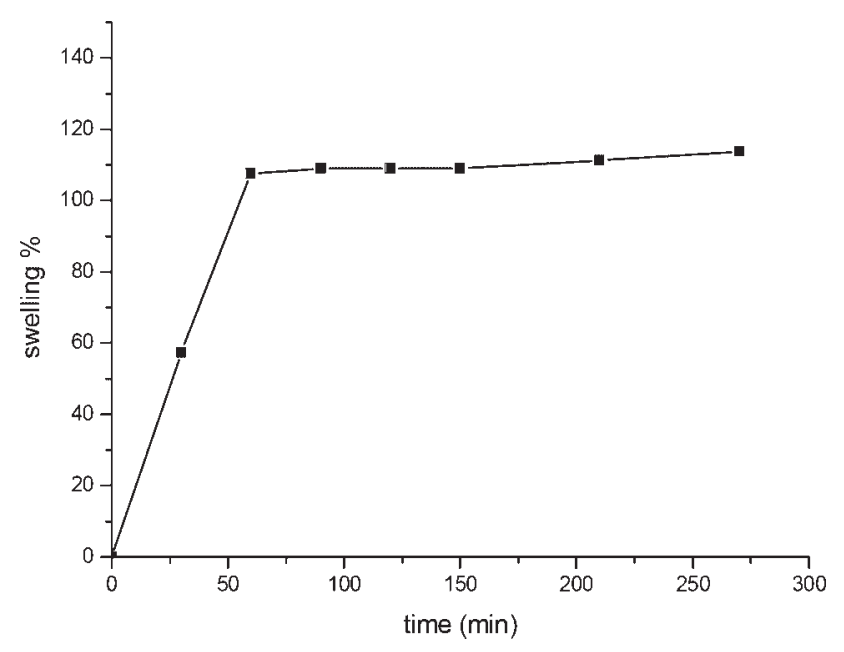

Figure 2. Swelling ratio vs. time for chitosan fibers. 
a)

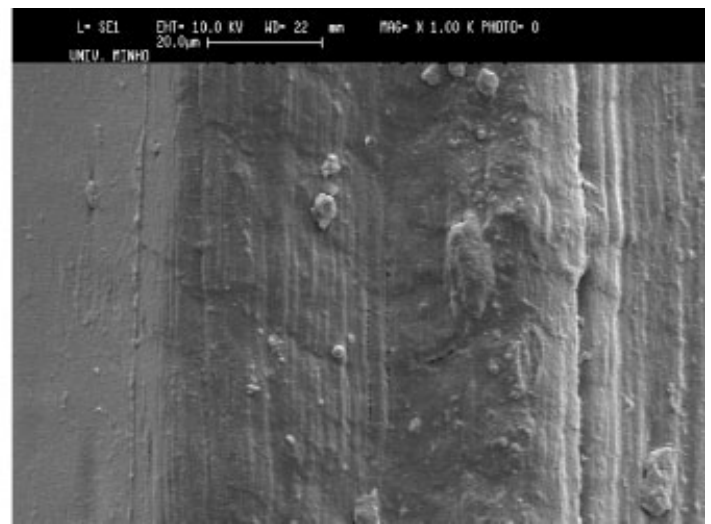

b)

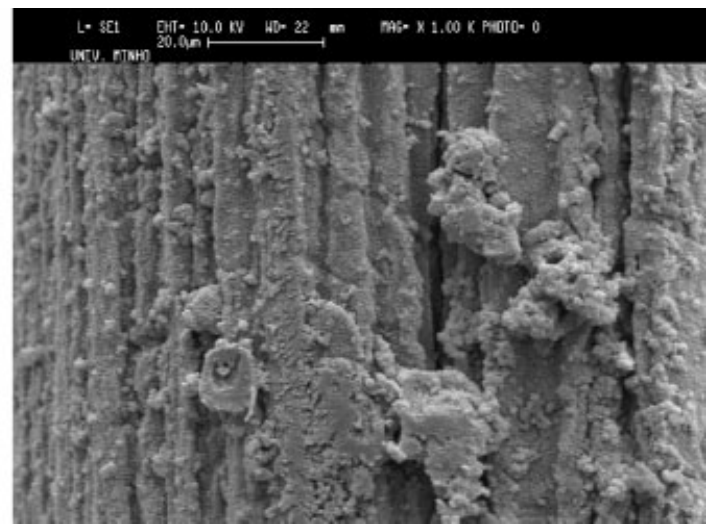

c)

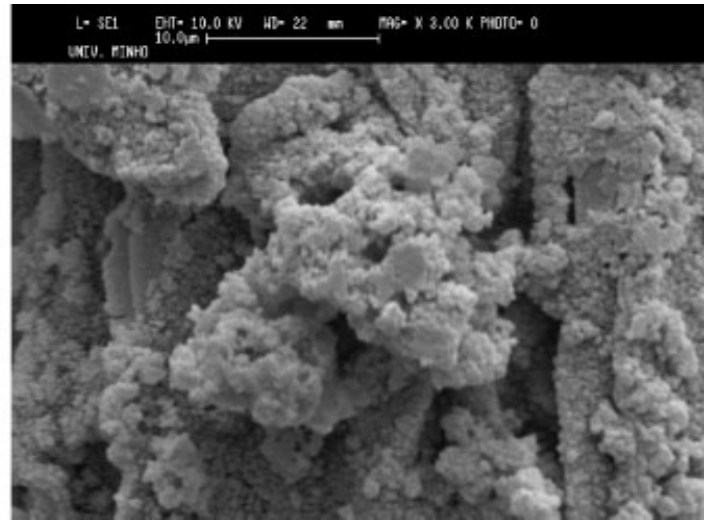

Figure 3. SEM micrographs of chitosan fibers; a) before, $b$ ) after $30 \mathrm{~d}$ immersion in $\mathrm{SBF}(\times 1000)$, c) after $30 \mathrm{~d}$ immersion in SBF $(\times 3000)$.

SBF solution after $14 \mathrm{~d}$ leading to the precipitation and growth of the Ca-P layer that was observed by SEM.

\section{Cytotoxicity Test}

Regarding the in vitro study of the cytotoxic effect of chitosan fibers; results indicate that the material passed the test with a cytotoxic index of 0 (see Table 3). This means that as for tissue culture polystyrene surfaces and UHMWPE, when cell culture media was replaced by

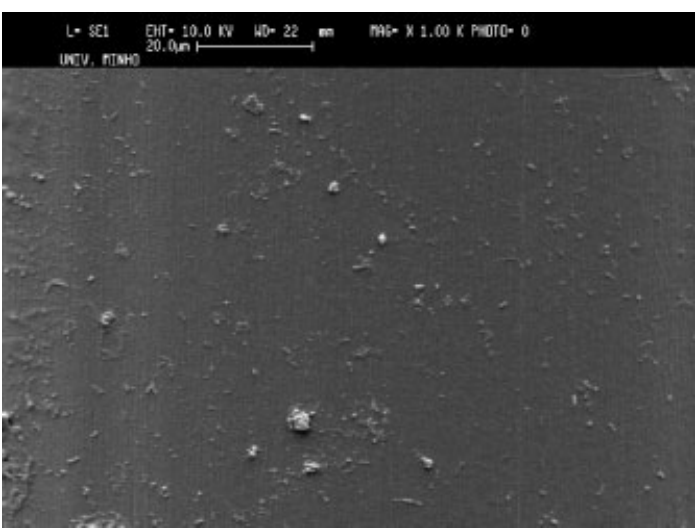

Figure 4. SEM micrographs of commercial available polyamide fibers after $30 \mathrm{~d}$ immersion in $\mathrm{SBF}(\times 1000)$.

extracts of materials in study, good fibroblast proliferation and no cell morphology modifications were observed. This is not typically for all of biodegradable polymers.

\section{Chitosan Fiber Meshes}

\section{Scanning Electron Microscopy}

SEM images of fiber meshes are presented in Figure 7a, b, c. As it can be seen in the micrograhps, the chitosan fibers could be formed into mesh structures having an average pore size in the range of $100-500 \mu \mathrm{m}$. In addition, it was possible to see that fibers have sticked to each other. This structure gives them good mechanical strength as well as it will enable cell ingrowth, as required for tissue engineering scaffolding applications.

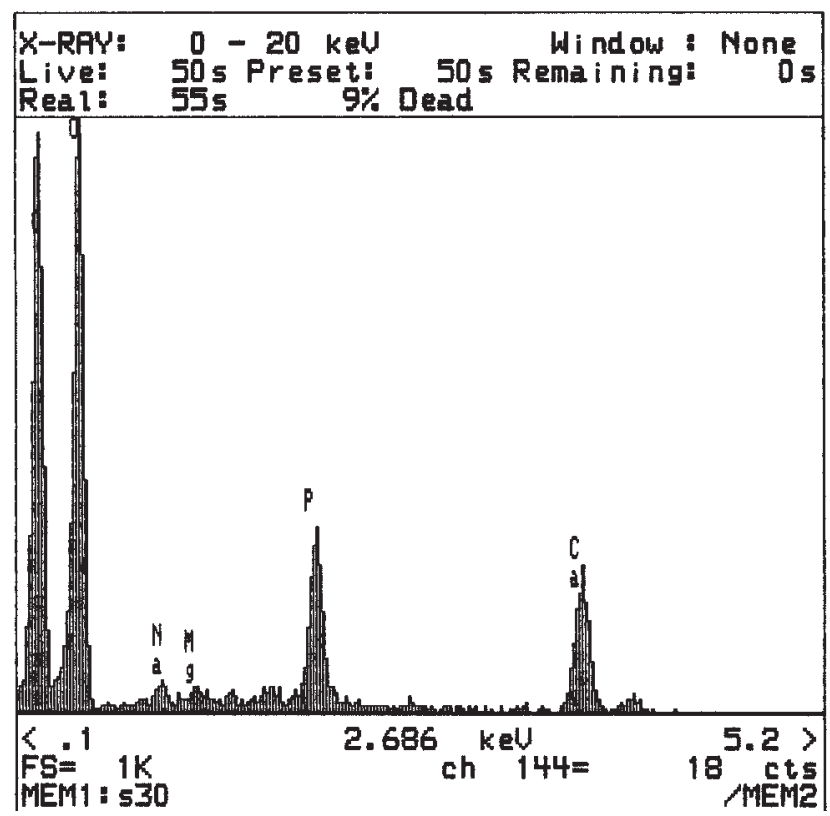

Figure 5. EDS spectra of the Ca-P coatings on the surface of chitosan fibers (after $30 \mathrm{~d}$ in SBF). 


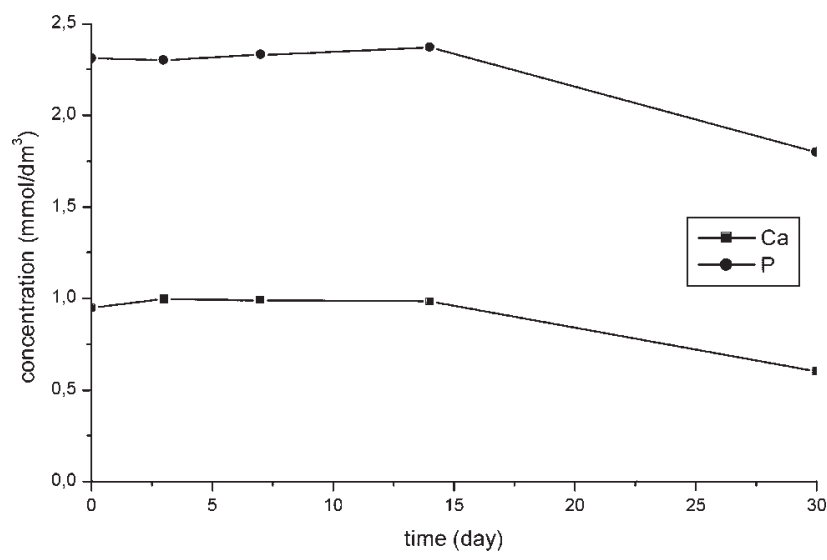

Figure 6. Evolution of the $\mathrm{Ca}$ and $\mathrm{P}$ elemental concentrations in the SBF as a function of immersion time of chitosan fibers in SBF.

\section{Mechanical Properties}

Swelling experiments are usually carried out by means of measuring the sample's weight after different periods of immersion. It is then very difficult to access the swelling data during short-time immersion periods, which would be rather relevant for materials having a fast swelling rate. The studied chitosan meshes are among such systems, where the equilibration of water content occurs mostly in the first hour after immersion. The innovative procedure described here in permits to follow this process by continuously monitoring the height of the samples immediately after the moment they are immersed. Obviously, considering an isotropic swelling, the height of the sample is easily correlated with the increase of volume.

The sample's strain $\varepsilon=\left(h-h_{0}\right) / h_{0}$, where $h$ and $h_{0}$ are the measured and initial heights of the sample) is measured against time. The results obtained with an initially dry sample with $h_{0}=2.351 \mathrm{~mm}$ are shown in Figure 8 . The increase of the strain is faster in the first $20 \mathrm{~min}$ and the limit strain seems to be less than $30 \%$.

The data in Figure 8 was fitted according to the stretched exponential function:

$$
\varepsilon(t)=\varepsilon_{\infty}\left[1-\exp \left(-(t / \tau)^{\beta}\right)\right]
$$

where $\varepsilon_{\infty}$ is the limit swelling strain, $\tau$ is a characteristic swelling time and $\beta$ is an exponent parameter that measures

Table 3. Results comparing fibroblasts cytotoxic response and reactivity to chitosan materials and controls.

\begin{tabular}{lclc}
\hline Sample & $\begin{array}{c}\text { Total cytotoxic } \\
\text { response }\end{array}$ & Reactivity & Pass/Fail \\
\hline Chitosan fiber & 0 & None & Pass \\
Chitosan fiber mesh & 0 & None & Pass \\
$\quad$ scaffolds & 0 & None & Pass \\
UHMWPE & 0 & None & Pass \\
TCPS & 8 & Severe & Fail \\
Latex & & & \\
&
\end{tabular}

a)

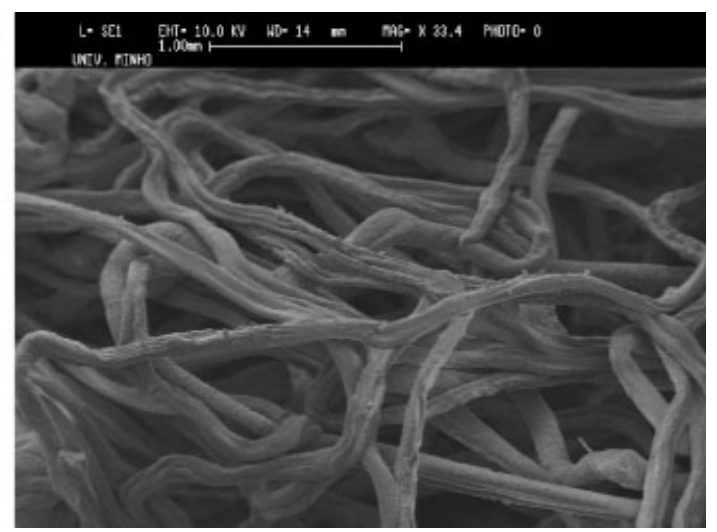

b)

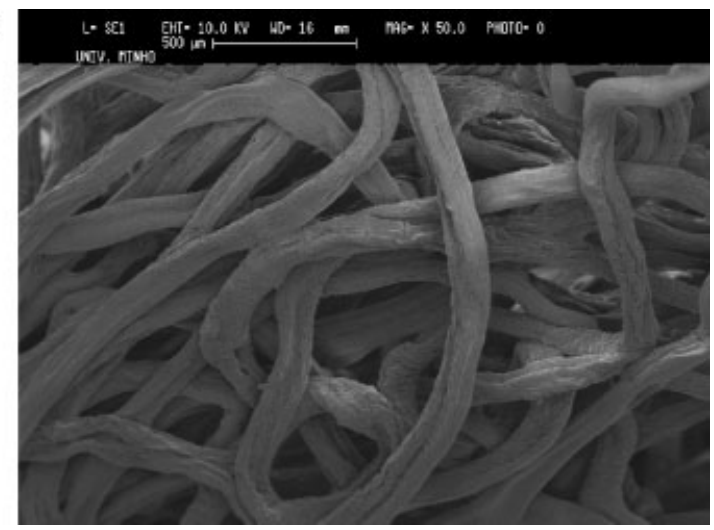

c)

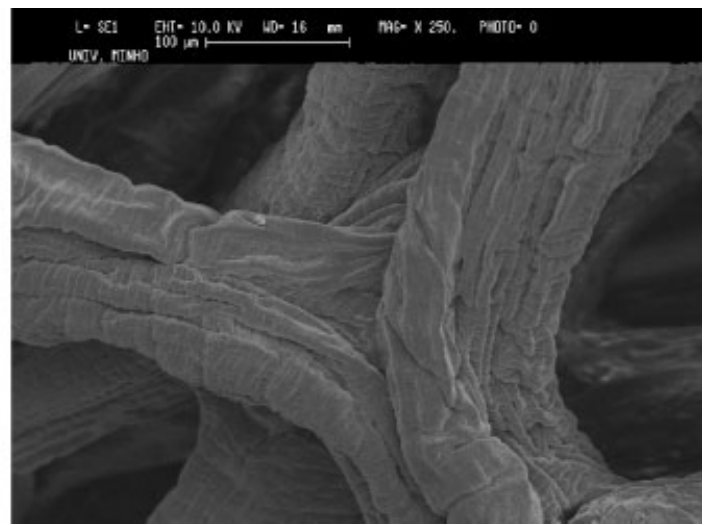

Figure 7. SEM micrographs of the structure of the developed chitosan fibers meshes; a) $\times 33.4$, b) $\times 50$, c) $\times 250$.

the deviation from a first-order swelling kinetics $(\beta=1)$. This function appears in this context as an empirical model that may be useful to characterize the strain at equilibrium and the swelling rate. This equation has often been used to follow relaxation processes, i.e., the isothermal evolution to the equilibrium when a system is subjected to an external perturbation (see, for example, ref. ${ }^{[31]}$ ); in that case the model has physical significance. The results in Figure 8 were fitted using a Levenberg-Marquard algorithm and the adjusted parameters were: $\varepsilon_{\infty}=29.4 \pm 0.08 \%, \tau=9.8 \pm$ $0.1 \mathrm{~min}$ and $\beta=0.54 \pm 0.003$. 


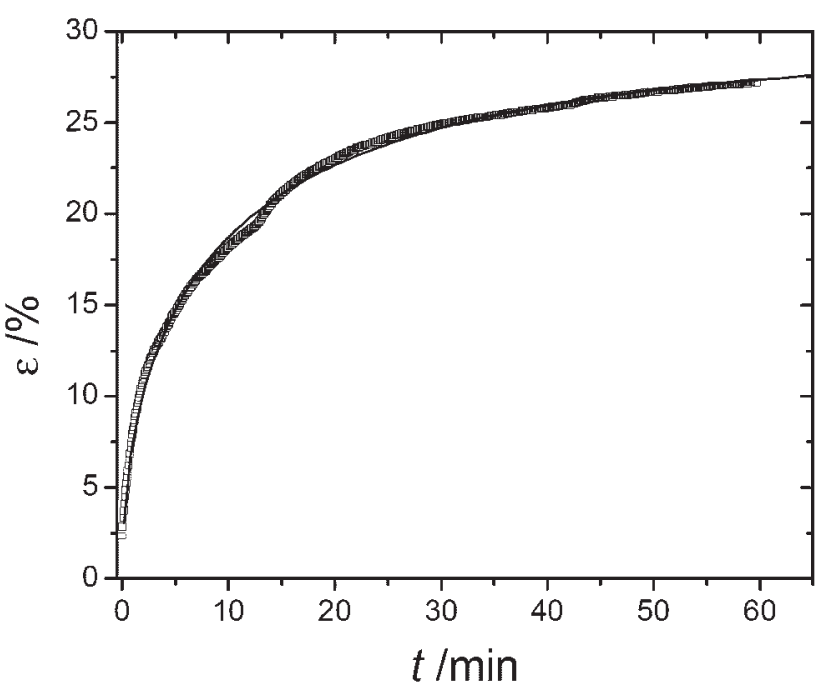

Figure 8. Evolution of the strain of a dry chitosan mesh after it was immersed at $37^{\circ} \mathrm{C}$ (symbols). The solid line corresponds to the best fit of the data according to Equation (2).

The meshes may be subjected to static loads if used in different biomedical applications. In polymer-based materials, an applied static stress usually results in a continuous, long term deformation under the applied stress, called creep. Such phenomenon may be relevant in the clinical use of the studied meshes, being associated with changes on the mechanical performance of the material and in variations in the dimensions of the implant. In this work creep/recovery of the immersed chitosan mesh was studied in simulated in vitro conditions.

The "instantaneous" increase of the strain is mainly associated with the expulsion of water within the mesh and thus is weakly assigned with the changes of geometry of the chitosan fibers, but rather is to the quantity of water initially in equilibrium and to the original cellular geometry of the sample. Please note that the initial strain value $(\approx 35 \%)$ is higher than the plateau in the swelling experiments $(\approx 30 \%$, as suggested in Figure 8). The excess strain $(\approx 5 \%)$ is then basically due to the deformation of the mesh geometry of the chitosan sample.

After the initial deformation a clear delayed response is observed in the creep experiment (see inset graphs in Figure 9). The approximately linear relationship found in the log time graphs may be in accordance with the powerlaw for the time dependence found in the viscoelastic creep response in another system. ${ }^{[32]}$ Therefore, it is possible to assign this delayed creep to the viscoelastic properties of the chitosan fibers.

The removing of the strain results in a quick decrease of strain that occurs with a similar time-scale of the swelling experiment $(\tau \approx 10 \mathrm{~min})$. Therefore, most of the strain recovery is related with the swelling of the mesh. After $1 \mathrm{~h}$ of recovery, it is found that the strain is far to converge to 0 .

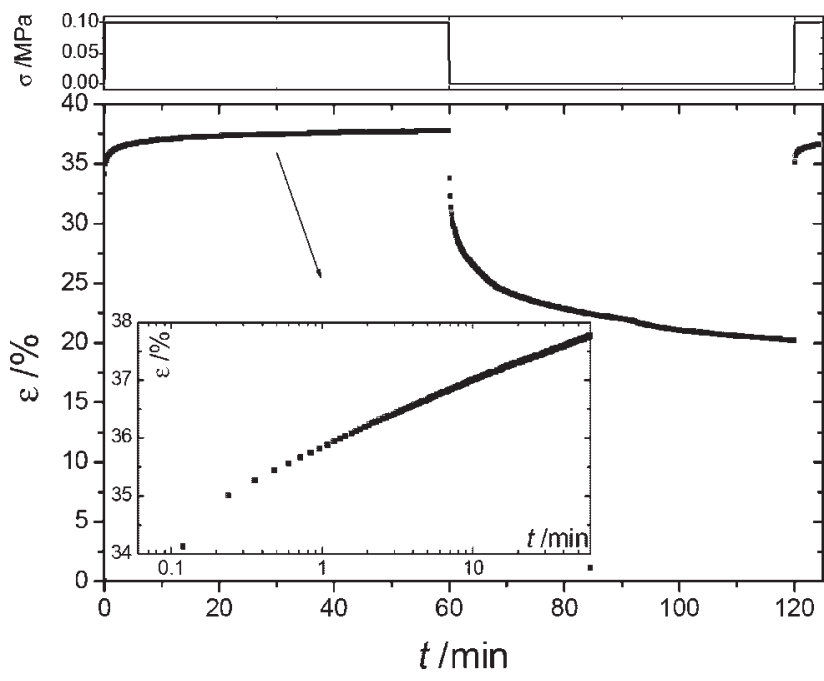

Figure $9 . \quad$ Creep $(60 \mathrm{~min})$ followed by a recovery stage $(60 \mathrm{~min})$ and, finally, a second short creep run performed at $37^{\circ} \mathrm{C}$ in the immersed chitosan mesh. The inset graph shows the viscoelastic component of the first creep run, in a log time scale.

This may be an indication that part of the mesh structure was irreversibly transformed during the creep state, leading to a more compact cellular arrangement.

After this creep/recovery experiment the sample was again subjected to the same creep stress. The strain appears to respond similarly to the first creep exposition, being an indication that neither apparent complex memory effects nor drastic structural changes occur in the sample.

The rheological behavior of the immersed mesh was also characterized by DMA. Both storage and loss moduli $\left(E^{\prime}\right.$ and $E^{\prime \prime}$ ) were measured at $37^{\circ} \mathrm{C}$ in the frequency range $0.5-10 \mathrm{~Hz}$, which are typical frequencies found in physiological stress-variation situations (blood pumping rate, masticatory frequency, time for patient movements. . .). The results are shown in Figure 10. It is to be noticed that the values may change if the experiment is carried out at different stresses, due to the variation of the water content in equilibrium inside the mesh. Please also note that $E^{\prime}$ and $E^{\prime \prime}$ may be transformed into other viscoelastic parameters, ${ }^{[33]}$ such as compliance, $D^{*}=D^{\prime}-\mathrm{i} D^{\prime \prime}=1 / E^{*}$, shear complex modulus, $G^{*}=G^{\prime}+\mathrm{i} G^{\prime \prime}=E^{*} /\left[2\left(1+v^{*}\right)\right]$, where $v^{*}$ is the complex Poisson's ratio, shear compliance, $J^{*}=J^{\prime}-\mathrm{i} J^{\prime \prime}=$ $1 / G^{*}$, or the real and imaginary components of the dynamic viscosity, $\eta^{\prime}=G^{\prime \prime} / \omega$ and $\eta^{\prime \prime}=G^{\prime} / \omega$.

The storage modulus increases with increasing frequency, $E^{\prime}$ being typically above $1 \mathrm{MPa}$. The loss modulus shows the inverse tendency. This results in a decrease of the loss factor, $\tan \delta=E^{\prime \prime} / E^{\prime}$ with increasing frequency (inset graphics in Figure 10). This parameter measures the fraction of the imposed mechanical stress that is dissipated in the form of heat. As $\tan \delta$ is typically above 0.15 for $f<1 \mathrm{~Hz}$, one may conclude that the damping capability of the system is significant, which may be important for some 


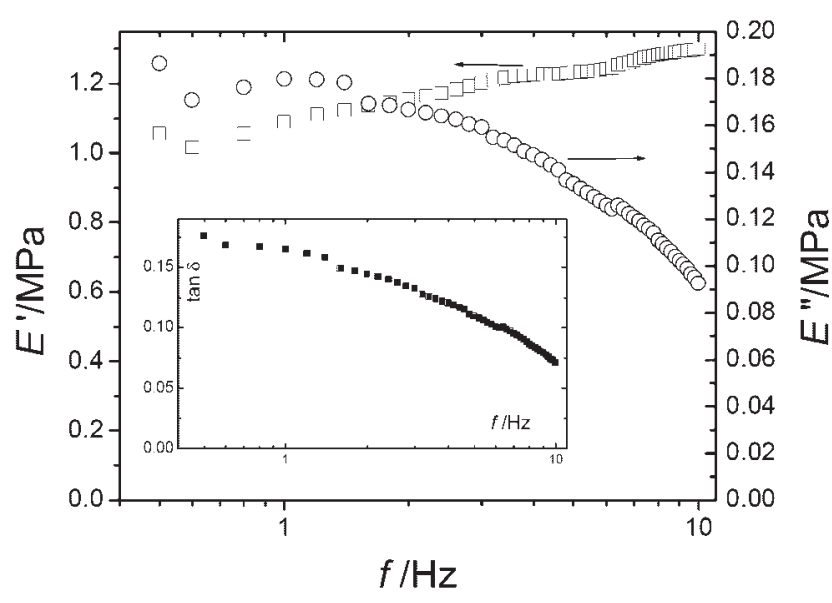

Figure 10. Storage and loss moduli $\left(E^{\prime}\right.$ and $\left.E^{\prime \prime}\right)$ as a function of frequency $(f)$ at $37^{\circ} \mathrm{C}$ of the immersed chitosan mesh. The inset graph shows the frequency dependence of the loss factor.

biomedical applications that require dissipation of mechanical energy. It was found that living cells behave as soft glassy materials existing close to a glass transition, implying that cytoskeletal proteins may regulate cell mechanical properties. ${ }^{[34]}$ This kind of evidences leads one to speculate on the existence of some advantages upon biological functions when the materials of the implant that will be in contact with living tissues, also present a viscoelastic character, as is the case for the studied chitosan mesh.

\section{Swelling Tests}

Figure 11 shows the swelling behavior of chitosan fiber meshes. In this Figure, it is possible to observe the typical swelling behavior of chitosan scaffolds. ${ }^{[7]}$ Samples reach the equilibrium water uptake ratio, which is around $170 \%$ $(\mathrm{w} / \mathrm{w})$, after about $3 \mathrm{~h}$ in $\mathrm{NaCl}$ solution. Although fiber meshes were swelled up to high ratio, they preserved their physical integrity upon incubation in aqueous solution. This property of chitosan fiber meshes may enable for

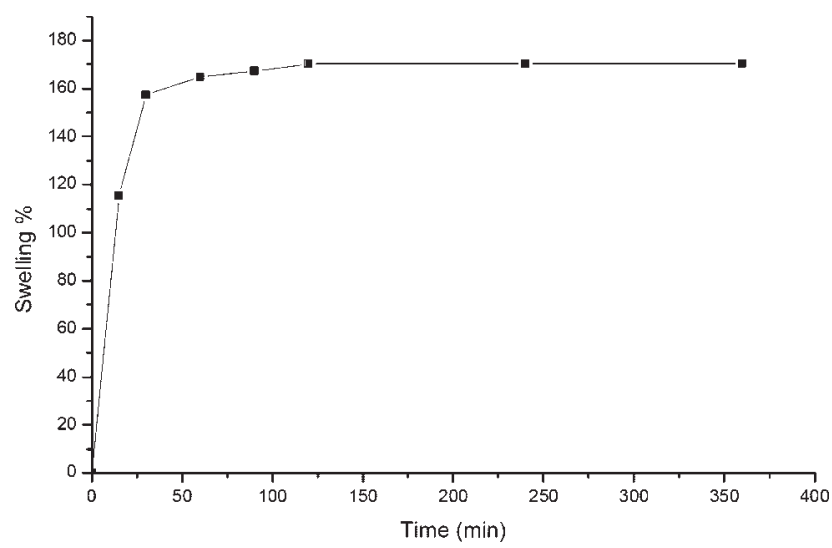

Figure 11. Swelling ratio vs. time for the developed 3-D chitosan fiber meshes. easy handling of the scaffold material in practical clinical applications. This result has been confirmed by DMA tests.

\section{Cytotoxicity and Biocompatibility: Direct Contact Test with Osteoblast-Like Cells}

Cytotoxicity test results for chitosan fiber mesh scaffolds can be seen in Table 3. They showed no cytotoxic effect, and behaved very similar to tissue culture and UHMWPE surfaces.

In order to observe cell morphology and proliferation of an osteoblast cell line over chitosan fiber mesh scaffold surfaces, SAOS-2 cells were directly cultured and incubated for 1 and 7 d. By means of observing Figure 12 and 13 it is possible to verify that scaffolds allowed for very significant cell proliferation from day 1 to day 7, respectively. After $7 \mathrm{~d}$, it was clearly seen that cells have been attaching to the scaffolds and bridging each other by means of filapodia structures which is typical for osteoblast-like cells (Figure 13). ${ }^{[35]}$ Furthermore, a detailed analysis of osteoblast-like cells morphology confirmed short-term cytotoxic results performed with fibroblasts, and allowed for observing extensive lamellipodium structure formation.

\section{Conclusions}

In this paper, attention has been focused on the preparation and properties of chitosan fiber and fiber meshes for potential use in tissue engineering applications. In the present study, chitosan fibers could be produced by a wet spinning technique. A subsequent drying treatment with methanol was used to improve the mechanical strength of the fibers. Mechanical tests showed that the fibers had enough tensile strength to be used to produce scaffolds with good mechanical performance. Unexpectedly, the developed fibers showed a bioactive behavior, which is also very important for biomaterials aimed to be used for instances in bone tissue engineering scaffolding. Fibers could also be formed

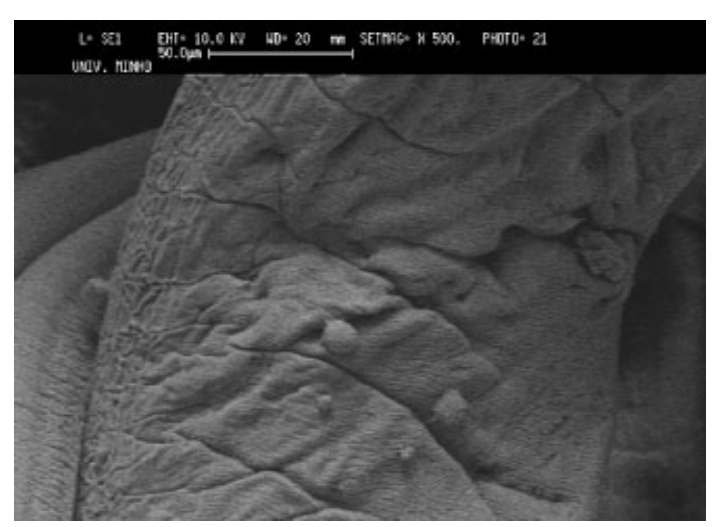

Figure 12. Osteoblast-like cells adhering to chitosan based fibers after $1 \mathrm{~d}$ of culture. 


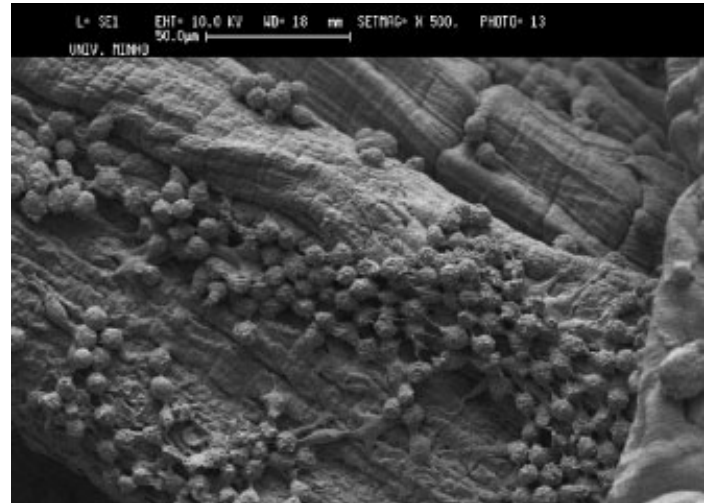

Figure 13. Osteoblast-like cells proliferating over chitosan based fibers after $7 \mathrm{~d}$ of culture.

into 3-D structure aimed to be used as tissue engineering scaffolds. One could observe that the mesh structure of the scaffold was suitable for cell ingrowth. This was confirmed by the results obtained on in vitro cell culture studies. Both types of sample extracts were comparable to UHMWPE clearly passing the test. In addition, osteoblasts growing over scaffold surfaces presented adequate morphology and good proliferation, proofing that the developed scaffolds might be used for bone tissue engineering applications.

Acknowledgement: K. Tuzlakoglu thanks the Portuguese Foundation for Science and Technology for providing her a $\mathrm{PhD}$ scholarship (SFRH/BD/8502/2002). This work was partially supported by FCT Foundation for Science and Technology, through funds from the POCTI and/or FEDER programmes.

[1] N. V. Majeti, R. Kumar, React. Funct. Polym. 2000, 46, 1.

[2] C. H. Lee, A. Singla, Y. Lee, Int. J. Pharm. 2001, 221, 1.

[3] T. Hayashi, Prog. Polym. Sci. 1994, 9, 663.

[4] D. Schaefer, I. Martin, P. Shastri, R. F. Padera, R. Langer, L. E. Freed, G. Vunjak-Novakovic, Biomaterials 2000, 21, 2599.

[5] G. Zund, S. P. Hoerstrup, A. Schoeberlein, M. Lachat, G. Uhlschmid, P. R. Vogt, M. Turina, Eur. J. Cardio-Thoracic Surg. 1998, 13, 160.

[6] U. Klinge, V. Schumpelick, B. Klosterhalfen, Biomaterials 2001, 22, 1415.

[7] E. B. Denkbas, M. Seyyal, E. Piskin, J. Membr. Sci. 2000, $4461,1$.

[8] J. K. Francis Suh, H. W. T. Matthew, Biomaterials 2000, 21, 2589 .
[9] H. Struszczyk, D. Wawro, A. Nicktaszcwicz, "Advances in chitin and chitosan", C. J. Brinc, P. A. Sandford, J. P. Zikakis, Eds., Elsevier, London 1992, p. 580.

[10] J.Z. Knaul, S. M. Hudson, K. A. M. Creber, J. Appl. Poly. Sci. 1999, 72, 1721.

[11] G. W. Urbanczyk, in: "Applications of Chitin and Chitosan", M. F. A. Gooden, Ed., Technomic, Lancaster 1997, p. 281.

[12] S. Hirano, K. Nagamura, M. Zhang, S. K. Kim, B. G. Chung, M. Yoshikawa, T. Midorikawa, Carbohydr. Polym. 1999, 38, 293.

[13] O. C. Agboh, Y. Qin, Polym. Adv. Technol. 1997, 8, 355.

[14] H. Zhang, Y. Du, J. Yu, R. Huang, L. Zhang, J. Appl. Polym. Sci. 2001, 80, 2558.

[15] J. Z. Knaul, M. Hooper, C. Chanyi, A. M. Creber, J. Appl. Polym. Sci. 1998, 69, 1435.

[16] J. Z. Knaul, S. M. Hudson, A. M. Creber, J. Appl. Polym. Sci. 1999, 72, 1721 .

[17] P. J. VandeVord, H. W. T. Matthew, S. P. DeSilva, L. Mayton, B. Wu, P. H. Wooley, J. Biomed. Mater. Res. 2002, 59, 585.

[18] A. Lahiji, A. Sohrabi, D. S. Hungerford, C. G. Frondoza, J. Biomed. Mater. Res. 2000, 51, 586.

[19] J. K. F. Suh, H. W. T. Matthew, Biomaterials 2000, 21, 2589.

[20] G. Kratz, C. Arnander, J. Swedenborg, M. Back, C. Falk, I. Gouda, O. Larm, Scand J. Plast. Reconstr Surg. Hand. Surg. 1997, 31, 119.

[21] S. Miyazaki, K. Ishii, T. Nadai, Chem. Pharm. Bull. 1981, 29, 3067.

[22] S. Miyazaki, H. Yamaguchi, M. Takada, W. M. Hou, Y. Takeichi, H. Yasubuchi, Acta Pharm. Nord. 1990, 2, 401.

[23] R. Muzzarelli, G. Biagini, A. Pugnaloni, O. Filippini, V. Baldassarre, C. Castaldini, C. Rizzoli, Biomaterials 1989, 10, 598.

[24] Y. L. Kuen, S. H. Wan, H. P. Won, Biomaterials 1995, 16, 1211.

[25] R. Muzzarelli, M. Mattioli-Belmonte, C. Tietz, R. Biagini, G. Ferioli, M. A. Brunelli, M. Fini, R. Giardino, P. Ilari, G. Biagini, Biomaterials 1994, 15, 1075.

[26] T. Kawakami, M. Antoh, H. Hasegawa, Y. Yamagishi, M. Ito, S. Eda, Biomaterials 1992, 13, 759.

[27] H. M. Kim, T. Furuya, T. Kokubo, T. Miyazaki, T. Nakamura, in: "Bioceramics 14", Palm Springs, USA 2001, p. 621.

[28] Y. Abe, T. Kokubo, T. Yamamuro, J. Mater. Sci. Mater. Med. 1990, 1, 233.

[29] I. B. Leonor, R. A. Sousa, A. M. Cunha, Z. P. Zhong, D. Greenspan, R. L. Reis, J. Mater. Sci. Mater. Med. 2002, 13, 10.

[30] ISO/EN 10993-5. Biological Evaluation of Medical Devices - Part 5: Tests for cytotoxicity, in-vitro methods: 8.2 tests on extracts.

[31] "Disorder effects on relaxational processes", R. Richert, A. Blumen, Eds., Springer-Verlag, Berlin 1994.

[32] L. F. Boesel, J. F. Mano, R. L. Reis, J. Mater. Sci. Mater. Med., submitted.

[33] J. D. Ferry, "Viscoelastic Properties of Polymers", $3^{\text {rd }}$ edition, Wiley, New York 1980.

[34] B. Fabry, G. N. Maksym, J. P. Butler, M. Glogauer, D. Navajas, J. J. Fredberg, Phys. Rev. Lett. 2001, 87, 14.

[35] Y. Wang, Q. Wu, G. Chen, Biomaterials 2004, 25, 669. 